\title{
Neutrino mass variability due to nonminimal coupling to spacetime curvature in neutrinophilic two-Higgs-doublet models
}

\author{
Roberto Onofrio ${ }^{1,2}$ \\ ${ }^{1}$ Dipartimento di Fisica e Astronomia "Galileo Galilei", \\ Università di Padova, Via Marzolo 8, Padova 35131, Italy \\ ${ }^{2}$ ITAMP, Harvard-Smithsonian Center for Astrophysics, 60 Garden Street, Cambridge, MA 02138, USA
}

(Dated: April 29, 2022)

\begin{abstract}
In neutrinophilic two-Higgs-doublet models, neutrinos acquire mass due to a Higgs field with vacuum expectation value of the order of $\simeq 10^{-2} \mathrm{eV}$, corresponding to a Compton wavelength in the $10 \mu \mathrm{m}$ range. This creates a situation in which nonminimal couplings between Higgs fields and spacetime curvature may lead to novel observable effects. Among these, we discuss the possibility of variable neutrino masses, with implications for the dependence of the neutrino oscillation frequency on the spacetime curvature, a further source of dispersion of the neutrino arrival times from supernovae events, and possibly also a mechanism leading to gravitationally-induced neutrino superluminality. Finally, we propose laboratory-scale experiments in which properly designed electroweak cavities may be used to change neutrino masses, which should be observable through time of flight experiments.
\end{abstract}

PACS numbers: 04.62.+v, 14.60.Pq, 14.80.Bn

The mass of elementary particles is, in the standard model, a dynamical observable depending on the vacuum expectation value (VEV) of the Higgs field and the Yukawa couplings. As such, it is possible in principle to change the mass by deforming the VEV of the Higgs field through macroscopic external fields. Since the Higgs has no coupling to the photons at tree level, this leaves open only the possibility of a nonminimal coupling to a strongcurvature region of spacetime. Unfortunately, due to the large mismatching between the typical space-time curvature lenghtscale and the Compton wavelength of the Higgs field, this possibility becomes realistic only in the presence of very large nonminimal couplings. This has been discussed in [1], leading to the prediction of Higgsrelated spectroscopic shifts due to changes of the electron mass in proximity of strong-gravity sources. The experimental observability of mass shifts at moderate values of the nonminimal coupling becomes more favourable if, as in various extensions of the standard model involving multiple Higgs doublets, some VEVs are intrinsically smaller. This occurs for instance in the context of the so-called neutrinophilic two-Higgs-doublet models [2, 3], in which neutrinos acquire mass through coupling to a Higgs doublet with VEV corresponding to a Compton wavelength of macroscopic size.

In this note, we discuss possible implications of such a Higgs doublet on the physics and astronomy of neutrinos. More in general, we want to focus the attention on a possibility that has not yet been explored to the best of our knowledge, i.e. that neutrinos, due to their intrinsically minute masses, are sensitive probes of the interplay between the curvature of spacetime and the Lagrangian of the standard model or its extensions. Then the following discussion has to be considered as a specific example of a more general framework, pioneered by Stodolsky [4], and still to be fully developed (see [5] for significant progress in this direction): as the precision of long baseline neutrinos experiments will increase, the standard model will be tested in a general Riemannian geometry, extending the realm of quantum field theory in curved spacetimes so far developed mainly for quantum electrodynamical phenomena involving photons [6].

We consider the Lagrangian density for two Higgs doublet fields in a generic curved spacetime in which a nonminimal coupling to the Ricci scalar $R$ is added to the standard model Lagrangian as

$$
\mathcal{L}_{\text {Higgs-Curvature }}=\xi_{R} R\left(\phi_{1}^{2}+\phi_{2}^{2}\right),
$$

where $\phi_{1}$ denotes the Higgs doublet of the standard model coupled to quarks and charged leptons, and $\phi_{2}$ is the Higgs doublet proposed in [2, 3], giving mass to neutrinos with Yukawa couplings comparable to the ones of the charged fermions and light quarks. The parameter $\xi_{R}$ measures the coupling strength between the Higgs fields and the curvature scalar $R$ and, to ensure compatibility with the equivalence principle, we assume that $\xi_{R}$ has the same value for the two Higgs doublets.

In the spontaneously broken phase, by denoting with $\mu_{i}$ and $\lambda_{i}$ the usual mass and self-interaction Higgs parameters (with $i=1,2$ for a two-Higgs-doublet model), the Higgs fields develop vacuum expectation values $v_{0}^{(i)}=$ $\left(-\mu_{i}^{2} / \lambda_{i}\right)^{1 / 2}$, which is equal to $v_{0}^{(1)}=250 \mathrm{GeV}$ for the Higgs doublet of the standard model, and $v_{0}^{(2)} \simeq 10^{-2}$ $\mathrm{eV}$ for the Higgs doublet only coupled to neutrinos 2, 3]. The presence of two VEVs differing by 13 orders of magnitude originates a hierarchy problem quite similar, even in quantitative terms, to the one generated in GUTs without supersymmetry, and recently the stability of quantum corrections has been studied in detail both for models containing Dirac and Majorana neutrino mass 
terms [7], and models considering only Dirac neutrinos [8].

As usual, the masses of the elementary particles are all directly proportional to the VEVs $v_{i}$ via the Yukawa coefficients of the fermion-Higgs Lagrangian density term, $m_{j}=y_{j} v_{1} / \sqrt{2}$ for charged leptons and for quarks of flavor $j$, and $m_{\nu}=y_{\nu} v_{2} / \sqrt{2}$ for the three neutrinos mass eigenstates. Hereafter we assume that the Yukawa couplings $y_{i}$ are of algebraic, rather than of dynamical character, and under this hypothesis the mass $m_{i}$ will be changed only due to changes in the Higgs VEVs. Therefore, in a curved spacetime the effective coefficient of the Higgs field $\mu_{i}^{2} \mapsto \mu_{i}^{2}+\xi_{R} R$, and the VEV of the Higgs fields will become spacetime dependent through the curvature scalar as $v_{0}^{(i)}=\sqrt{-\left(\mu_{i}^{2}+\xi_{R} R\right) / \lambda_{i}}$. If we assume a bare neutrino mass in flat spacetime $m_{\nu}^{(0)}=$ $y_{\nu} \sqrt{-\mu_{2}^{2} /\left(2 \lambda_{2}\right)}$, in the presence of curved spacetime its value will become

$$
m_{\nu}=\frac{y_{\nu}}{\sqrt{2}} \sqrt{-\frac{\mu_{2}^{2}+\xi_{R} R}{\lambda_{2}}}=m_{\nu}^{(0)} \sqrt{1+\xi_{R} R \Lambda_{2}^{2}}
$$

where we have introduced the reduced Compton wavelength associated to the Higgs mass parameter $\mu_{2}$ as $\Lambda_{2}=\hbar /\left(\mu_{2} c\right)$. Assuming a Higgs mass of value comparable to $v_{2} \simeq 10^{-2} \mathrm{eV}$, we obtain a Compton wavelength for the Higgs mass parameter $\Lambda_{2} \simeq 2 \times 10^{-5} \mathrm{~m}$. Notice that, under the hypothesis that the Higgs-curvature coupling strength is universal, all neutrino flavors undergo the same mass shift in the presence of a given gravitational source.

We complement this analysis by also discussing the case of a coupling of the Higgs field to the curvature via another invariant, such as the Kretschmann invariant defined as $K=R_{\mu \nu \rho \sigma} R^{\mu \nu \rho \sigma}$, where $R^{\mu \nu \rho \sigma}$ is the curvature tensor. This invariant plays an important role in quadratic theories of gravity [9 11] and has been already used in [1] to estimate putative Higgs shifts arising from atoms in proximity of spherically symmetric astrophysical strong-gravity sources, and in 12] to discuss limits coming from violations to the superposition principle for gravitational forces in the weak-field limit. In the weakfield limit the Higgs-curvature Lagrangian term will be written as

$$
\mathcal{L}_{\text {Higgs-Curvature }}=\xi_{K} \Lambda_{\mathrm{Pl}}^{2} K\left(\phi_{1}^{2}+\phi_{2}^{2}\right),
$$

where $\Lambda_{\mathrm{Pl}}=\left(G \hbar / c^{3}\right)^{1 / 2}$ is the Planck length, whose value is $\Lambda_{\mathrm{Pl}} \simeq 10^{-35} \mathrm{~m}$ in conventional quantum gravity models or $\Lambda_{\mathrm{Pl}} \simeq 10^{-19} \mathrm{~m}$ in models with early unifications of gravity to the other fundamental interactions via extra dimensions 13. By replacing the Ricci scalar coupling in Eq. (2) with the Kretschmann scalar coupling we obtain

$$
m_{\nu}=\frac{y_{\nu}}{\sqrt{2}} \sqrt{-\frac{\mu_{2}^{2}+\xi_{K} \Lambda_{\mathrm{Pl}}^{2} K}{\lambda_{2}}}=m_{\nu}^{(0)} \sqrt{1+\xi_{K} \Lambda_{\mathrm{Pl}}^{2} K \Lambda_{2}^{2}}
$$

Since the Kretschmann invariant is nonzero outside a massive source, in this case we expect mass modulation even outside the mass, although with an amplitude quickly fading away from the source. However, due to the suppression due to the presence of the Planck length, the values of $\xi_{K}$ necessary to reproduce mass modulations of amplitude comparable to the one of the Ricci coupling are much larger, i.e. $\xi_{K}>>\xi_{R}$.

It may be worth to remark that under proper combinations of signs for $\xi_{R}$ and the Ricci scalar $R$, a curved spacetime with enough coupling to the Higgs field may yield a sign inversion in the argument of the square root in Eq. 2 and therefore an imaginary mass, leading to a transition from bradyonic to tachyonic behavior for neutrinos. Models for tachyonic neutrinos have been already envisaged several decades ago [14 17], and very recently several contributions have appeared with a variety of mechanisms to accommodate the earlier OPERA outcome in a variety of scenarios (see [18] for a review). While the OPERA result has been lately both retracted after identifying a systematic effect and shown to be incompatible with the one obtained in the contiguous ICARUS detector at LNGS [19], it may be still premature to definitely rule out the possibility that neutrinos are particles endowed with a superluminal behavior 20]. Previous results as the one reported by the MINOS collaboration, $\delta_{c}=(v-c) / c=(5.1 \pm 2.9) \times 10^{-5}$ (at $68 \%$ confidence level) [21] and, at a weaker confidence level, various evidences for a negative central value of the muonic neutrino mass from precision studies of the pion decay 22 27], show that superluminal neutrino propagation is still not ruled out at a high level of confidence. In any event, gravitationally-induced superluminality is potentially expected to play a role only inside or near strong-gravity sources, ruling out the possibility of terrestrial experiments for its verification. For instance, the Ricci scalar can be simply estimated inside a neutron star in the hypothesis of homogeneous mass distribution. For a neutron star of mass $M_{n}=2 M \odot$ and radius $r_{n}=12$ $\mathrm{Km}$, the Ricci scalar is $R_{n}=6 G M_{n} /\left(c^{2} r_{n}^{3}\right) \simeq 10^{-8} \mathrm{~m}^{-2}$. Then the superluminal regime occurs inside the neutron star if $\xi_{R}$ is negative and larger in absolute value than $\left|\xi_{R}\right|>2.5 \times 10^{17}$. This value of nonminimal coupling is much smaller than the one assumed in [28, 29] in the framework of a proposal for the Higgs field as responsible for inflation, in which a value $\xi_{R} \simeq 10^{4}$ in units for which $\hbar=c=1$, corresponding to $\xi=10^{4}(c / \hbar)^{2}=8 \times 10^{88}$ using MKSA units for $\hbar$ and $c$, was discussed. For fermions other than neutrinos, coupled to the large VEV Higgs doublet of the standard model, it is easy to verify that the mass shift in the presence of spacetime curvature in- 
side a neutron star is negligible under any reasonable assumption unless an unrealistically large $\xi_{R}$ is assumed, since its effectiveness scales as the ratio of the squared Compton wavelengths of the Higgs doublets which is, in turn, proportional to the ratio between the VEVs of the two doublets, $v_{1} / v_{2} \simeq 2.5 \times 10^{13}$.

We envisage at least two frameworks in which a possible non-minimal coupling between the second Higgs doublet and the spacetime curvature could give rise to observable effects. First, since the discussed mechanism applies identically to all neutrino flavors, we expect that the square mass difference is also changed in the presence of a non-zero Ricci scalar, and this induces a modulation of the oscillation parameter for the $\nu_{i} \longleftrightarrow \nu_{j}$ oscillation $\Delta m(i j)^{2}=\Delta m(i j)_{0}^{2}\left(1+\xi R \Lambda_{2}^{2}\right)$ which depends on Earth's parameters such as its radius and mass, unlike the oscillation parameter $\Delta m(i j)_{0}^{2}$ related to the neutrinos bare masses. In principle solar neutrino oscillations could also be affected by this effect, resulting in a contribution to the day-night measurements already performed [30], but it is easy to show that this is negligible for a broad range of nonminimal couplings due to the independence of the measurable average survival probability upon the source-detector distance. Second, neutrinos created during supernovae events, depending on the location of their production into the collapsing core, will be also affected by this mechanism. Neutrinos generated closer to the core center will experience larger mass shifts with respect to the one originated in the outer layers, and this will imply a further spreading in the arrival time on terrestrial detectors. In this regard, the time lapse between neutrino events detected in different laboratories during SN 1987A 31] could be reanalyzed through this mechanism of mass dispersion. Furthermore, we expect a large spreading during the stage of abrupt truncation of the neutrino flux occurring in the early stage of formation of the black hole possibly resulting from the core-collapse supernova 32].

Finally, we briefly discuss possibility of modulating the mass via properly tailored structures. The Compton wavelength of the second Higgs doublet falls in the micrometer to hundred micrometers range, depending on its VEV conjectured to be in the $10^{-3}-10^{-1} \mathrm{eV}$ range. One may therefore envisage extended structures made of many parallel layers of material spaced by comparable distances which should suppress the propagation of the Higgs mode in the space in between, analogously to the well-known inhibition of electromagnetic modes in a conducting cavity [33, 34]. In the region occupied by this structure the neutrinos will propagate as massless or lighter particles, depending on the degree of cavityinduced suppression of the VEV of the second Higgs doublet. Interposing this structure along the path of a neutrino beam would therefore allow for the detection of a smaller time of flight with respect to the free propagation with full action of the second Higgs doublet. If the sup- pression factor in the electroweak cavity for antineutrinos differs from the one for neutrinos, a comparison between muonic neutrinos and antineutrinos beams should also result in different times of flight.

In conclusion, we have identified a mechanism leading to a variability of the neutrino mass in the presence of non-minimal coupling to curvature invariants of a light Higgs doublet already considered in various extensions of the standard model. The existence of this mechanism does not seem to clash with any experiment or observation available so far, stressing the importance of systematically exploring bounds on the curvature coupling constant to the Ricci scalar $\xi_{R}$ or to the Kretschmann invariant $\xi_{K}$. Possible tests of this effect have been qualitatively discussed such as atmospheric neutrino oscillations, the identification of a further source of spreading for the time of flight originated in the SN 1987A supernova event, and the possibility of gravitationally-induced superluminality inside strong-gravity astrophysical objects. As a further test unrelated to the presence of spacetime curvature, we have proposed engineered electroweak cavities able to suppress the Higgs propagation, which could allow to modulate the time of flight of freely propagating neutrinos as in the CERN-LNGS experiments.

[1] R. Onofrio, Phys. Rev. D 82, 065008 (2010).

[2] F. Wang, W. Wang, J.M. Yang, Europhys. Lett. 76, 388 (2006).

[3] S. Gabriel, S. Nandi, Phys. Lett. B 655, 141 (2007).

[4] L. Stodolsky, Gen. Rel. Grav. 11, 391 (1979).

[5] D. V. Ahluwalia and C. Burgard, Gen. Rel. Grav. 28, 1161 (1996); Y. Grossman and H.J. Lipkin, Phys. Rev. D 55, 2760 (1997); D. Píriz, M. Roy, and J. Wudka, Phys. Rev. D 54, 1587 (1996); G.M. Fuller and Y.-Z. Qian, Nucl. Phys. A 606, 167 (1996); C. Y. Cardall and G.M. Fuller, Phys. Rev. D 55, 7960 (1997).

[6] N.D. Birrell, P.C.W. Davies, Quantum Fields in Curved Space, Cambridge University Press, Cambridge, 1982.

[7] N. Haba, T. Horita, Phys. Lett. B 705, 98 (2011).

[8] T. Morozumi, H. Takata, and K. Tamai, Phys. Rev. D 85, 055002 (2012).

[9] S. Deser, P. van Niewenhuizen, Phys. Rev. D 10, 401 (1974).

[10] K.S. Stelle, Phys. Rev. D 16, 953 (1977).

[11] F.W. Hehl, Y. Ne'eman, J. Nitsch, P. von der Heyde, Phys. Lett. B 78, 102 (1978).

[12] R. Onofrio, Eur. Phys. J. C 72:2006 (2012).

[13] N. Arkani-Hamed, S. Dimopoulos, and G. Dvali, Phys. Lett. B 429, 263 (1998); Phys. Lett. B 436, 257 (1998); Phys. Rev. D 59, 086004 (1999).

[14] R. Mignani, E. Recami, Riv. Nuovo Cimento 4, 209 (1974).

[15] A. Chodos, A.I. Hauser, V.A. Kostelecký, Phys. Lett. B 150, 431 (1985).

[16] E. Giannetto, G.D. Maccarrone, R. Mignani, E. Recami, Phys. Lett. B 178, 115 (1986).

[17] R.J. Hughes, G.J. Stephenson Jr., Phys. Lett. B 24, 95 
(1990).

[18] B.-Q. Ma, Mod. Phys. Lett. A 27, 1230005 (2012).

[19] M. Antonello et al. (ICARUS Collaboration), Phys. Lett. B 713, 17 (2012).

[20] The window of opportunity for superluminal propagation of neutrinos is now severely constrained through the recent result from the ICARUS collaboration in which an r.m.s. value of the average of the recorded distribution for the time of flight delay between photons and neutri$\operatorname{nos}$ is $\delta t=$ tof $_{\gamma}-$ tof $_{\nu}=(0.3 \pm 4.0$ (stat.) \pm 9.0 (sys.) $)$ ns over an expected time of flight for the photons of $(2439098 \pm 1.5) \mathrm{ns}$ [19].

[21] P. Adamson et al. (MINOS Collaboration), Phys. Rev. D 76, 072005 (2007).

[22] E.V. Shrum, K.O.H. Ziok, Phys. Lett. B 37, 114 (1971).

[23] G. Backenstoss et al., Phys. Lett. B 43, 539 (1973).

[24] D.C. Lu et al., Phys. Rev. Lett. 45, 1066 (1980).
[25] H.B. Anderhub et al., Phys. Lett. B 114, 76 (1982).

[26] R. Abela et al., Phys. Lett. B 146, 431 (1984).

[27] B. Jeckelmann et al., Phys. Rev. Lett. 56, 1444 (1986).

[28] F. Bezrukov, M. Shaposhnikov, Phys. Lett. B 659, 703 (2008).

[29] F. L. Bezrukov, A. Magnin, M. Shaposhnikov, Phys. Lett. B 675, 88 (2009).

[30] Q.R. Ahmad et al., Phys. Rev. Lett. 89, 011302 (2002).

[31] W. D. Arnett, J. N. Bahcall, R. P. Kirshner, and S. E. Woosley, Annu. Rev. Astron. Astrophys. 27, 629 (1989).

[32] J. F. Beacom, R. N. Boyd, and A. Mezzacappa, Phys. Rev. Lett. 85, 3568 (2000); Phys. Rev. D 63, 073011 (2001).

[33] E. M. Purcell, Phys. Rev. 69, 681 (1946).

[34] D. Kleppner, Phys. Rev. Lett. 47, 233 (1981). 